\title{
Transmission of Hepatitis C Virus Infection to Tree Shrews
}

\author{
Zhi-Chun Xie, ${ }^{*}$ Jose-Ignacio Riezu-Boj, ${ }^{2}$ Juan-Jose Lasarte, ${ }^{2}$ Javier Guillen, ${ }^{3}$ \\ Jie-Han Su,* Maria-Pilar Civeira, ${ }^{2}$ and Jesus Prieto ${ }^{2,1}$

\begin{abstract}
*Department of Preventive Medicine, Guangxi Medical University, Nanning, Guangxi 530021, People's Republic of China; and ${ }^{2}$ Department of Internal Medicine and ${ }^{3}$ Animal Services, Faculty of Medicine, University of Navarra, Pamplona, Spain
\end{abstract}

Received December 22, 1997; returned to author for revision February 3, 1998; accepted March 4, 1998

\begin{abstract}
Although hepatitis $\mathrm{C}$ virus ( $\mathrm{HCV}$ ) infection can be reproduced in chimpanzees, these animals are rare and expensive. Tree shrews (tupaias) are small animals, closely related to primates, which adapt easily to a laboratory environment. In this work we have investigated the susceptibility of Tupaia belangeri chinensis to HCV infection. Tupaias caught in the wild in Yunnan (China) were inoculated in China with HCV genotype $1 \mathrm{~b}$ (study A) and in Spain with a mixture of genotypes 1b, 1a, and 3 (study B). In study B tupaias were divided into three groups: group I was inoculated without previous manipulation, group II received 750 cGy of X-ray whole-body irradiation before inoculation, and group III was used as control. Transient or intermittent viremia occurred in $34.8 \%(8 / 23)$ and anti-HCV in $30.4 \%(7 / 23)$ of tupaias in study A. In study B a transient viremia was detected in $20 \%(2 / 10)$ in group I and in $50 \%(2 / 4)$ in group II. Anti-HCV was found in 1 tupaia from group I and in 3 from group II. Viremia lasted for longer and anti-HCV tended to reach higher titers in animals which received total body irradiation. ALT elevations and nonspecific pathological changes occurred in inoculated tupaias; however, the wild nature of the animals precludes the interpretation of these changes as solely due to $\mathrm{HCV}$ infection. In summary our results show that $\mathrm{T}$. b. chinensis are susceptible to HCV and that whole-body irradiation may possibly increase the efficiency of the infection. These animals may serve as an in vivo system for culturing $\mathrm{HCV}$ and addressing pathophysiological and therapeutic issues of $\mathrm{HCV}$ infection. c 1998 Academic Press
\end{abstract}

Key Words: Tupaia; irradiation; PCR; ALT; antibodies.

\section{INTRODUCTION}

Hepatitis $\mathrm{C}$ virus $(\mathrm{HCV})^{2}$ infection is a major cause of chronic hepatitis, liver cirrhosis, and liver cell cancer worldwide (Schreier et al., 1996; Zhang et al., 1990; Alter et al., 1990). Since the cloning of HCV in 1989 (Choo et al., 1989), progress in the knowledge of the basic biology, pathogenic mechanisms, and therapy of hepatitis $C$ has been hampered by the lack of an appropriate animal model (Abe et al., 1993). Although chimpanzees are easily infected with HCV and develop acute or chronic hepatitis (Alter et al., 1978; Bradley et al., 1979), these animals are rare, expensive, and difficult to handle and thus inappropriate for use in research programs requiring an abundant number of animals. Therefore, the finding of a small animal, easy to breed and of low cost, which would be susceptible to HCV infection, would be of great importance in facilitating progress in HCV research.

We acknowledge the financial help of J. Vidal, J. Soriano, and M. Mendez.

${ }^{1}$ To whom reprint requests should be addressed at Department of Internal Medicine and Liver Unit, Clônica Universitaria, Universidad de Navarra, Apartado 4209, 31080 Pamplona, Spain. Fax: + 48-296500. E-mail: jprieto@cun.unav.es.

${ }^{2}$ Abbreviations used: HCV, hepatitis C virus; PCR, polymerase chain reaction; HGV, hepatitis $G$ virus; $A L T$, alanine aminotransferase.
Tree shrews (Tupaia) are small squirrel-like mammals which live in Southeast Asia (Wang et al., 1991). The family Tupaiidae has been included either in the order Insectivora or in the order Primates, but more recently tree shrews are being considered in a single order called Scandentia (Martin, 1990). They have a relatively restricted geographic distribution, including southern China, Vietnam, Laos, Kampuchea, India, Thailand, Malaysia, Indonesia, Philippines, Burma, Bangladesh, and Bhutan (Martin, 1990). Tupaia includes eleven species; among these, $T$. belangeri has the widest distribution. Eight $\mathrm{T}$. belangeri subtypes are found in China: T. b. yaoshanensês, T. b. gaoligongensis, T. b. lepcha, T. b. tonquinia, T. b. chinensis, T. b. yunalis, T. b. modesta, and T. b. assamensts (Wang et al., 1991). Tree shrews have shown susceptibility to infection by human rotavirus (Wan et al., 1982), herpes simplex virus type 1 and 2 (Darai et al., 1978), and human hepatitis viruses A, B, and D (Li et al., 1995; Walter et al., 1996; Yan et al., 1996a,b; Zan et al., 1981). Susceptibility of Tupaia to HCV infection has not been reported. We tested this possibility in two transmission experiments using Chinese tree shrews. This paper shows that tupaias can be infected in vivo with HCV and that whole-body irradiation might facilitate HCV infection of these animals. 
TABLE 1

Results of Serum HCV RNA and Anti-HCV in Tupaias from Study A

\begin{tabular}{|c|c|c|c|c|c|c|c|c|c|c|c|c|}
\hline \multirow[b]{2}{*}{ Animal } & \multicolumn{12}{|c|}{ Weeks postinoculation } \\
\hline & 0 & 2 & 5 & 7 & 9 & 14 & 18 & 23 & 27 & 31 & 36 & 47 \\
\hline $1^{\mathrm{a}}$ & - & - & - & - & - & - & - & - & - & nd & - & - \\
\hline $2^{\mathrm{a}}$ & - & - & - & - & - & - & nd & _- & - & - & - & - \\
\hline 4 & - & nd & - & - & - & - & - & - & + & - & - & + \\
\hline 5 & - & - & + & + & - & - & - & - & nd & - & - & - \\
\hline 6 & - & - & - & - & - & - & - & - & - & - & - & - \\
\hline 7 & - & - & - & - & - & - & nd & - & - & - & - & - \\
\hline 8 & - & - & nd & - & + & - & - & - & - & - & - & - \\
\hline 9 & - & + & + & + & - & + & - & - & + & - & - & - \\
\hline 10 & - & - & - & - & - & - & - & - & - & - & - & - \\
\hline 11 & - & - & - & + & - & nd & - & + & - & - & - & - \\
\hline 12 & - & - & - & - & - & - & - & - & - & - & - & - \\
\hline 13 & - & - & + & - & - & - & - & + & nd & - & - & - \\
\hline 14 & - & - & - & - & - & - & - & - & - & - & - & - \\
\hline 15 & - & - & - & - & - & - & - & - & - & - & - & - \\
\hline 16 & - & - & - & + & - & - & - & - & + & - & - & - \\
\hline 17 & - & - & - & - & - & - & - & - & - & - & - & - \\
\hline 18 & - & - & - & - & - & - & - & - & - & - & - & - \\
\hline 19 & - & nd & - & - & - & - & - & - & nd & - & - & - \\
\hline 20 & - & - & - & - & - & - & - & - & - & - & - & - \\
\hline 21 & - & - & - & - & - & - & - & - & - & - & - & - \\
\hline 22 & - & nd & - & nd & - & - & nd & - & - & - & - & - \\
\hline 23 & - & + & - & - & - & - & - & - & - & - & - & - \\
\hline 24 & - & - & - & - & - & - & - & - & - & - & - & - \\
\hline 25 & - & nd & - & - & - & nd & - & - & - & - & - & - \\
\hline 26 & - & nd & - & - & - & - & - & - & - & - & - & - \\
\hline
\end{tabular}

Note. nd, not done. (+) Indicates positive HCV RNA and (-) negative HCV RNA. The shaded area indicates positive anti-HCV.

${ }^{a}$ Control animals.

\section{RESULTS}

\section{Study A}

HCV RNA and anti-HCV were negative in all preinoculation blood samples obtained from experimental animals and in the two control tupaias. Eight inoculated animals (34.8\%) developed HCV viremia, as determined by PCR at different times during the 47 weeks of follow-up (Table 1). HCV RNA was first detected 2 weeks postinoculation in two animals (Nos. 9 and 23). The viremia was transient in two tupaias and intermittent in six. In tupaia N 0. 9 HCV RNA was detected in serum from weeks 2 to 7 postinoculation, it became negative on week 9 , and reappeared transiently on weeks 14 and 27.

Anti-HCV was found transiently in serum in seven (30.4\%) tupaias (Table 1). In three animals (Nos. 19, 22, and 23) the antibody persisted for 1 to 2 months. The fact that in 6 animals anti-HCV appeared after several weeks of seronegativity indicates that a carrying over of antibodies of the inoculum was not responsible for seropositivity. As seen in Table 1 three tupaias tested positively for both anti-HCV and HCV RNA at different time points during evolution but only two serum samples (one from animal No.9 and the other from No.13) were positive simultaneously for the two determinations. Anti-HCV was also found in four animals (Nos. 7, 19, 22, and 26) that never exhibited detectable viremia.

Peaks of transaminases (in most cases between weeks 7 and 18) were observed in five inoculated tupaias (Fig. 1A) and in one control animal. In all tupaias with increased serum transaminases HGV RNA determinations were performed, the results being negative in all animals tested. On week 50 postinoculation, liver biopsy specimens were obtained from four tupaias with previous elevation of ALT and/or detectable anti-HCV antibody (Nos. 13, 19, 22, and 24). The pathological examination showed ballooning degeneration of hepatocytes in all these animals, together with multinucleated liver cells and macrovesicularor focal necrosis with inflammatory infiltrate in some of them. One granulomatous lesion probably associated with parasite infection was also observed in one animal. The wild nature of the tupaias precludes the interpretation of biochemical and pathological changes as the solely due to HCV infection.

\section{Study B}

HCV RNA and anti-HCV antibodies were negative in all serum samples from the control group and in all prein- 


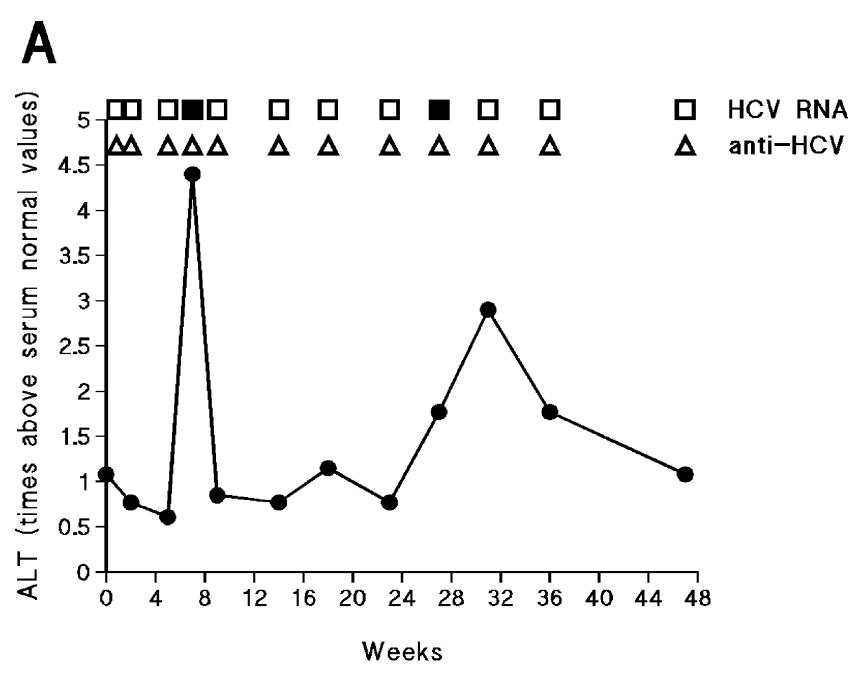

B

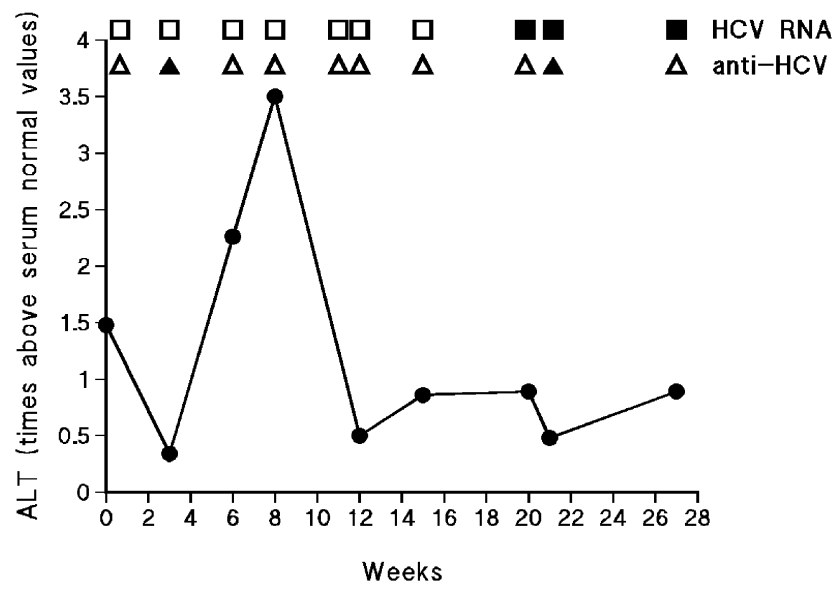

FIG. 1. Evolution of ALT levels and serological HCV markers in two representative tupaias from study A (tupaia No. 16, A) and study B (tupaia No. 19, B). Solid and open squares represent, respectively, positive and negative assays for HCV RNA. Solid and open triangles represent positive and negative anti-HCV, respectively.

oculation samples from groups I and II (Table 2). In contrast, HCV RNA became positive in two tupaias from group I (20\%) and in half of the animals (tupaias No. 7 and 19) from group II (Table 2). It was interesting to observe that while HCV RNA could be detected only for a brief period in group I, the viremia lasted longer in animals from group II. Positive viremia obtained with primers from the $5^{\prime}$ untranslated region was confirmed with primers from the core region (Table 2). HCV genotyping and quantification of HCV RNA were performed in three of the animals with positive viremia (Nos. 14, 7, and 19) (Table 3). We found genotype $1 \mathrm{~b}$ in all infected animals and coinfection $1 b / 3$ in one of them. The viral titer was low in all the infected tupaias, ranging from less than $10^{4}$ to $3.5 \times 10^{5}$ copies per milliliter of serum $(\mathrm{c} / \mathrm{ml})$.

Liver specimens from seven animals (three from group I, three from group II, and one from group III) were processed for detection of HCV RNA. As shown in Table 2, HCV RNA could be detected in liver tissue in two tupaias from group II. The positive result obtained with primers from the $5^{\prime}$ noncoding region was confirmed with primers from the core region of HCV. Viral loads in the liver of tupaias No. 7 and 19 were $10^{5}$ and less than $10^{3}$ copies/ $\mu$ g of RNA, respectively (Table 3 ).

Anti-HCV antibody appeared transiently in three animals and in a recurring manner in one. In three cases we found coincidence of positivity for anti-HCV and HCV RNA. In one case viremia was not accompanied by seropositivity and, conversely, anti-HCV developed in an animal that never presented positive viremia. Interestingly, while anti-HCV was detected in only one tupaia from group I, it was found in three of four animals from group II. In one of these tupaias (No. 19, week 21) the optical density in the ELISA test reached considerably high values (Table 2). Analysis of antigenic specificities using RIBA test showed that anti-C 33 c was the earliest and most frequent antibody (Table 4).

A transient peak of ALT elevation occurred in five animals from group I and in one from group II. The peak occurred between weeks 8 and 12 in most cases (Fig 1B). No significant ALT changes were observed in control animals. Testing for HGV RNA was performed in serum samples from animals showing elevated ALT at any time during the follow-up. In all cases HGV RNA was negative. Liver biopsy was obtained in all animals from the three groups on week 27 postinoculation. Histological examination of liver tissue in infected animals showed the presence of ballooning degeneration of hepatocytes, multinucleated liver cells, and fatty degeneration in most of these tree shrews. None of these changes could be interpreted as the sole result of HCV infection since multinucleated hepatocytes, fatty degeneration, and/or focal necrosis with inflammatory infiltrates were found in three animals from the control group. Granulomas possibly related to parasite infection were found in two tupaias from group I and in one from group III.

Liver biopsy was repeated on week 33 in two animals from group II with positive serum HCV RNA (tupaias No. 7 and 19) and in two control tupaias (No. 11 and 20) and the samples were processed for electron microscopy. Ultrastructural examination of the liver showed fat droplets, hyperplasia of smooth endoplasmic reticulum, and multiple membranous arrays resembling myelin figures. These changes were not found in tupaias from the control group. We did not find the typical cytoplasmic tubular structures described in chimpanzees with experimental HCV (Bradley et al., 1981; Shimizu et al., 1979).

\section{DISCUSSION}

In this paper we report the results of two studies which are concordant in showing that $\mathrm{T}$. b. chinensis can be 
TABLE 2

Results of Serum and Liver HCV RNA and Serum Anti-HCV Antibodies in Tupaias from Study B

Serum

Liver

Weeks postinoculation

\begin{tabular}{|c|c|c|c|c|c|c|c|c|c|c|c|c|}
\hline & 0 & 3 & 6 & 8 & 11 & 12 & 15 & 20 & 21 & 27 & 27 & 33 \\
\hline Animal & $5^{\prime a} \mathrm{C}$ & $5^{\prime} \mathrm{C}$ & $5^{\prime} \mathrm{C}$ & $5^{\prime} \mathrm{C}$ & $5^{\prime} \mathrm{C}$ & $5^{\prime} \mathrm{C}$ & $5^{\prime} \mathrm{C}$ & $5^{\prime} \mathrm{C}$ & $5^{\prime} \mathrm{C}$ & $5^{\prime} \mathrm{C}$ & $5^{\prime} \mathrm{C}$ & $5^{\prime} \mathrm{C}$ \\
\hline 1 & - & - & - & - & - & - & - & - & nd & - & - & nd \\
\hline 2 & - & - & - & - & - & - & - & - & nd & nd & nd & nd \\
\hline 3 & - & - & - & - & - & - & - & -- & - & - & nd & nd \\
\hline 4 & - & - & - & - & - & - & - & -- & - & - & nd & nd \\
\hline 5 & - & - & - & - & - & - & - & - & nd & - & nd & nd \\
\hline 12 & - & - & - & - & ++ & - & - & - & - & - & - & nd \\
\hline 13 & - & - & - & - & - & - & - & - & - & - & nd & nd \\
\hline 14 & - & - & - & ++ & - & - & - & -- & - & - & - & nd \\
\hline 16 & - & - & - & - & - & - & - & - & nd & - & nd & nd \\
\hline 17 & - & - & - & - & - & - & - & - & nd & - & nd & nd \\
\hline $7^{b}$ & - & - & ++ & - & - & -- & -- & ++ & +- & ++ & ++ & - \\
\hline $8^{b}$ & - & - & - & - & - & - & - & - & nd & - & nd & nd \\
\hline $15^{\mathrm{b}}$ & - & - & - & - & - & - & - & - & - & - & - & nd \\
\hline $19^{b}$ & - & -- & - & -- & -- & - & -- & ++ & ++ & +- & - & ++ \\
\hline $10^{c}$ & - & - & - & - & - & nd & - & - & nd & - & - & nd \\
\hline $11^{c}$ & - & - & - & - & - & nd & - & - & nd & - & nd & nd \\
\hline $20^{c}$ & - & - & - & - & - & nd & - & -- & nd & - & nd & nd \\
\hline $21^{\mathrm{c}}$ & - & - & - & - & - & nd & - & - & nd & _- & nd & nd \\
\hline
\end{tabular}

Note. nd, not done. (+) Indicates positive HCV RNA and (-) negative HCV RNA. The shading area indicates positive anti-HCV; optical density of ELISA: tupaia No. 15 (week 3) =0.291; tupaia No. 19 (week 3) =0.357; tupaia No. 7 (week 6) =0.303; tupaia No. 14 (week 8) =0.230; and tupaia No. 19 (week 21) $=1.418$.

${ }^{a} \mathrm{HCV}$ region of primers; 5', 5' untranslated region; $\mathrm{C}$, core region.

b Irradiated animals.

${ }^{c}$ Control animals.

infected in vivo with HCV. Whether other subtypes of T. belangeri are also susceptible to this infection is not known. In fact attempts in our laboratory to transmit HCV to tupaias with their origin in Thailand and bred in a European primate center failed to produce positive results (unpublished observations).

In this work rigorous criteria have been adopted to classify serum samples as HCV RNA positive, namely,

TABLE 3

Viral Load and HCV Genotypes in Infected Tupaias from Study B

\begin{tabular}{ccccc}
\hline Tupaia No. & Week & Genotype & $\begin{array}{c}\text { Viral load } \\
\text { in serum }\end{array}$ & $\begin{array}{c}\text { Viral load } \\
\text { in liver }\end{array}$ \\
\hline 14 & 8 & $1 b$ & $3,5 \times 10^{5}$ & nd \\
7 & 6 & $1 b$ & $3,5 \times 10^{5}$ & nd \\
& 20 & $3-1 b$ & $<10^{4}$ & nd \\
19 & 27 & nd & nd & $10^{5}$ \\
& 20 & $1 b$ & nd & nd \\
& 21 & 1b & $3 \times 10^{5}$ & nd \\
& 33 & nd & nd & $<10^{3}$ \\
\hline
\end{tabular}

Note. nd, not done.

${ }^{\text {a }}$ Copies/ml of serum.

${ }^{b}$ Copies/ug RNA. the inclusion of multiple negative controls in each PCR run and the need for repeated positivity in two valid assays. In addition, PCR positivity was ascertained in several specimens using primers from the core region of HCV genome. Thus, there seems to be little risk of false positivities in our PCR findings.

Our data show that viremia occurred in $34.8 \%$ of tupaias from study A, inoculated in China with genotype $1 \mathrm{~b}$. In study B conducted in Spain, the proportion of nonmanipulated tupaias which developed viremia after inoculation with a mixture of genotypes was only $20 \%$. The reason for this difference in the percentage of infected

TABLE 4

Analysis of Antigenic Specificities in Anti-HCV-Positive Sera from Tupaias (Study B) Using RIBA HCV 3.0

\begin{tabular}{ccccccc}
\hline & & \multicolumn{6}{c}{ Antigens used in RIBA HCV 3.0 } \\
\cline { 3 - 7 } Tupaia No. & Week & C100 & C33 & C22 & NS5 & SOD \\
\hline 14 & 8 & \pm & + & - & - & - \\
15 & 3 & - & \pm & - & - & - \\
19 & 3 & - & + & - & - & - \\
& 21 & \pm & + & - & $2+$ & - \\
\hline
\end{tabular}


tupaias is not clear since the animals used in the two studies come from the same geographical area (Yunnan, China). It may be possible that HCV quasispecies of the Chinese inocula adapt better to tupaia biology than the quasispecies contained in the European inocula or that the amount of virus in the Chinese inocula was higher (unfortunately viral load in the latter could not be determined). Alternatively, the mixture of genotypes in the inocula used in study B might unfavourably affect the replication of the eventually dominant genotype. In fact, although in this study the inoculum contained genotypes $1 b, 1 a$, and 3 , genotype $1 b$ was found to thrive better in tree shrews.

In study B we attempted to increase the efficiency of $\mathrm{HCV}$ transmission by subjecting animals to total body irradiation. We found that half of the surviving irradiated tupaias developed viremia and that HCV RNA tended to persist for longer in these tree shrews than in their nonirradiated mates. Moreover, while HCV RNA could not be detected in liver tissue from nonmanipulated tupaias, it was present in the liver of two irradiated animals. These findings indicate that irradiation, probably through its immunosuppressive effect, may enhance HCV replication in tree shrews. Our results open the way to explore other maneuvers which might facilitate HCV replication in tupaias, thus increasing the value of these animals as an experimental in vivo system of HCV infection.

In addition to developing viremia, tupaias also produce anti-HCV antibodies. In keeping with HCV RNA results, the prevalence of anti-HCV was highest in tree shrews from study A and in group II animals from study B. One of the irradiated tupaias developed very high titers of anti-HCV at week 21 postinoculation in coincidence with a rebound of HCV replication (Tables 2 and 4). In this case antibodies were directed against NS5 $(2+)$ and $C 33 \mathrm{C}(1+)$. This was an exception since, in agreement with the chimpanzee model (Shindo et al., 1992), the latter antigen was the main target of anti-HCV antibodies in most of the analyzed animals. It should also be noted that tupaias, similarly to chimpanzees, do not produce anti-C22 in response to HCV infection (Shindo et al., 1992).

As mentioned before (see Results) the time pattern of presentation of anti-HCV in inoculated tupaias indicates that seropositivity is not a consequence of carrying over of antibodies from the inoculum. The long delay in the appearance of anti-HCV in some cases suggests that these antibodies are the result of ongoing HCV replication. It is intriguing, however, that some seropositive animals never exhibited detectable HCV RNA. Hence, it seems possible that HCV replication may occur in some tupaias at an undetectable level which could nevertheless be sufficient to induce a humoral response. In fact, in samples where HCV RNA could be quantitated, the level of viremia was found to be very low, equal to or below $3.5 \times 10^{5}$ copies per $\mathrm{ml}$, which is considerably inferior to the level $\left(10^{7} \pm 10^{9}\right)$ found in patients with chronic hepatitis C (Larrea et al. 1996; Gavier et al., 1997).

Histopathological changes, mainly hepatocellular ballooning, fat degeneration, and the presence of multinuclear hepatocytes, occurred in many of the tree shrews which received HCV-positive inocula, but some of these morphological alterations were also observed in the control group. Occasional granulomas suggesting parasitic infection were found in some control and HCV-inoculated tupaias. It should be noted that the tree shrews used in the present study were captured in the jungle and wild tupaias are known to be frequently infected by viruses, such as tree shrew herpes virus, which may cause spontaneous hepatitis (Wu et al., 1983). For similar reasons variations in transaminase levels should be interpreted with caution. We observed significant ALT elevations in 11 tupaias which received HCV-positive inocula and only in one control animal. The ALT peak took place in most of the animals between the 8th and 12th weeks, which is the approximate time of occurrence of the transaminase peak in the acute phase of experimental HCV infection in chimpanzees (Bradley et al., 1990; Farci et al., 1991).

On electron microscopy, tupaias with positive viremia exhibited microvesicular fat, abnormal cytoplasmic vesiculation with hypertrophy of smooth endoplasmic reticulum, but not the classical multilaminated tubular structures observed in the chimpanzee model (Bradley et al., 1981; Shimizu et al., 1979). These alterations were not found in control tupaia livers. However, whether the changes observed in the infected tupaias are related to HCV infection could not be established in the present study.

To summarize, this report shows that $T$. b. chinensis is susceptible to HCV infection and from our preliminary data it seems that susceptibility to this infection could possibly be increased by whole-body irradiation. The small size of tupaias, their relatively low cost, their easy adaptation to a laboratory environment, and their high reproductive activity in captivity make this animal a possible in vivo system for culturing HCV which may be valuable in testing antiviral agents and addressing relevant pathophysiological issues of HCV infection.

\section{MATERIAL AND METHODS}

Animals

Adult tree shrews, subtype T. b. chinensis, captured in the wild in Yunnan Province, China, were used in two different study protocols ( $A$ and $B$ ). All animals were negative for hepatitis $B$ virus markers.

In study A, 25 tree shrews (10 male and 15 female; body weight $105.7+159.4 \mathrm{~g}$, mean, $121.4 \mathrm{~g}$ ) were kept in the Institute for Research on Zoology, Guangxi Medical University (Yunnan, China). They were housed in steel cages at a temperature of $23 \pm 2^{\circ} \mathrm{C}$, relative humidity of 
$70 \pm 80 \%$ and a 12 -h light/12-h dark photo cycle. Animals were fed basal diet supplemented with milk, eggs, and fruits. Water was supplied ad libitum.

In study B, 20 tree shrews (10 male and 10 female) were imported from China and kept at the animal facilities of the University of Navarra (Pamplona, Spain). Animals shrews (body weight $124.9+169.6 \mathrm{~g}$, mean, $143.0 \mathrm{~g}$ ) were housed individually in steel cages at $23 \pm 2^{\circ} \mathrm{C}$, relative humidity $50 \pm 70 \%$, and a 12/12-h light/dark cycle; they were fed commercial tree shrew chow ad libitum (Altromin, Germany) and fruits. They had free access to water.

\section{Inoculation and study protocol}

Study A. A pool of serum from four Chinese patients with chronic hepatitis $C$ (one case of posttransfusion hepatitis, one case of chronic hemodialysis, and two cases of community-acquired hepatitis) was used for inoculation. The inoculum was HCV RNA and anti-HCV positive and negative for hepatitis $B$ virus markers (HBsAg, Anti-HBs, HBeAg, anti-HBe, and anti-HBC). The $\mathrm{HCV}$ genotype of the inoculum was $1 \mathrm{~b}$. Virus concentration in the inoculum was not determined. Animals were inoculated intravenously with $0.15 \mathrm{ml}$ of serum and the same volume of saline was administered to two animals which served as controls (one male and one female). Blood was drawn before and 2, 5, 7, 9, 14, 18, 23, 27, 31, 36 , and 47 weeks after inoculation. Each time, $1 \mathrm{ml}$ of blood was collected from the thigh vein and serum samples were distributed in three aliquots for each animal. One was immediately used for alanine aminotransferase (ALT) determination and the other two were stored at $-40^{\circ} \mathrm{C}$ until used (for anti-HCV and HCV RNA determinations). In the 50th week postinoculation, surgical liver tissue samples were obtained from four animals with a previous elevation of ALT and/or detectable anti-HCV antibody. Liver biopsies were fixed in $10 \%$ Formalin and embedded in paraffin.

Study B. The inoculum consisted of a pool of the same amount of serum from five Spanish patients with chronic hepatitis C. The HCV genotype and virus concentration of these cases were as follows: patient 1 , genotype $1 b$, concentration $5 \times 10^{7} \mathrm{c} / \mathrm{ml}$; patient 2 , genotype $1 \mathrm{~b}$, concentration $8 \times 10^{6} \mathrm{c} / \mathrm{ml}$; patient 3 , genotype $1 \mathrm{~b}$, concentration $1.2 \times 10^{7} \mathrm{c} / \mathrm{ml}$; patient 4, genotype 1a, concentration $1 \times 10^{9} \mathrm{c} / \mathrm{ml}$; and patient 5, genotype 3 , concentration $1.1 \times 10^{9} \mathrm{c} / \mathrm{ml}$. All sera were negative for hepatitis $B$ virus markers (HBsAg, anti-HBs, $\mathrm{HBeAg}$, anti-HBe, and anti-HBC). In this study protocol animals were divided into three groups: group I (10 animals: 5 male and 5 female) was inoculated without previous irradiation, group II ( 6 animals: 3 male and 3 female) received 750 cGy of X-ray whole-body irradiation 2 days before inoculation, and group III (4 animals: 2 male and 2 female) which were used as control. Two animals from group II died after irradiation, and thus this group was reduced to only 4 tupaias. Animals from groups I and II were inoculated three times during the first week ( 2 days interval between inoculations) with $0.4,0.8$, and $0.8 \mathrm{ml}$ of the inoculum. Group III received normal human serum instead of the HCV-positive inoculum with the same volume and time schedule as the other two groups.

Blood samples were collected before and at $3,6,8,11$, $12,15,20,21$, and 27 weeks after inoculation. Each time, 2 to $3 \mathrm{ml}$ of blood was collected from the thigh vein and serum was aliquoted and stored at $-80^{\circ} \mathrm{C}$.

In the 27th week of follow-up, surgical liver biopsy was performed in all animals and in the 33rd week in selected cases. Liver samples were divided into three parts; one was fixed in $10 \%$ Formalin and then embedded in paraffin, and the other two were embedded in OCT (Sakura Finetek Europe, Zoeterwoude, Netherlands) and kept at $-80^{\circ} \mathrm{C}$ for RNA extraction. Surgical liver biopsy was also performed in eight additional, not inoculated, tree shrews. For electron microscopy liver biopsies were fixed for $6 \mathrm{~h}$ at $4^{\circ} \mathrm{C}$ in $4 \%$ glutaraldehyde in $0.1 \mathrm{M}$ sodium cacodylate buffer at pH 7.2 and postfixed in 1\% phosphate-buffered osmium tetroxide. After dehydration in ethanol and washing in propylene oxide, the tissue fragments were embedded in Epon 812. Semithin sections, 1 $\mu \mathrm{m}$ in thickness, were cut, mounted on glass slides, stained with methylene blue, and studied by light microscopy. For electron microscopy, thin sections were double stained with uranyl acetate and lead hydroxide and observed with a Zeiss $10 \mathrm{CR}$ electron microscope.

\section{Biochemical and serological determinations}

ALT activity was determined using the Reitman \pm Frankel method in study $A$ and by the Wroblewski and LaDue procedure using a commercially available kit (Sigma Diagnostic, St. Louis, M O) in study B. ALT activity was considered significantly elevated if higher than 2.5 times the mean preinoculation ALT values.

To determine the presence of anti-HCV antibodies, a passive hemagglutination assay (Abbott HCV PHA second generation; Dainabot Co., Tokyo, Japan) was employed in study $\mathrm{A}$. Both structural and nonstructural antigens of HCV (C100-3, pHCV31, and pHCV34) were included in this assay. The test was performed according to manufacturer's instructions. Samples were considered positive when hemagglutination occurred at $2^{-5}$ dilution or higher. In study B, anti-HCV antibodies were assayed using a third generation ELISA (Ortho, Raritan, NJ) according to manufacturer's instructions.

HCV RNA was determined in serum or liver tissue using a reverse transcription $\neq$ polymerase chain reaction (PCR) as described elsewhere (Gil et al., 1993). Briefly, an Ultraspect RNA isolation system (Biotecx Lab, Houston, TX) was used to extract RNA from $100 \mu$ l of serum. RNA was subsequently reverse-transcribed, followed by 30 cycles of PCR amplification using a set of primers from 
the $5^{\prime}$ noncoding region of the HCV genome (outer primers: sense, 5'-CCT GTG AGG AAC TAC TGT CT-3'; antisense, 5'CTA TCA GGC AGT ACC ACA AG-3'; $95^{\circ} \mathrm{C} 15 \mathrm{~s}$ for denaturation, $52^{\circ} \mathrm{C} 12 \mathrm{~s}$ for annealing, and $72^{\circ} \mathrm{C} 20 \mathrm{~s}$ for extension). One microliter of the PCR product was then subject to 30 cycles of amplification as described for the first PCR with a set of inner primers (sense, 5'-ACT GTC TTC ACG CAG AAA GC-3'; anti-sense, 5'GAC CCA ACA CTA CTC GGC TA-3'). In each run of PCR, eight serum samples and three negative controls were tested. Multiple negative controls were included to detect contamination. The assay was considered valid only if all of the negative controls were negative. To further confirm our results, samples were taken as positive only if the positive result was repeated in two valid assays. In addition, in study $B$ samples which were PCR positive using primers from the $5^{\prime}$ noncoding region of the $\mathrm{HCV}$ genome were retested with two sets of primers derived from the core region (Viazoz et al., 1994). The two steps of amplification reactions (35 cycles each) were performed according to the following profile: denaturation, $95^{\circ} \mathrm{C} 15 \mathrm{~s}$; annealing $50^{\circ} \mathrm{C} 12 \mathrm{~s}$; extension, $72^{\circ} \mathrm{C} 20 \mathrm{~s}$.

The quantification of HCV RNA was carried out using a competitive $P C R$ procedure as previously reported (Larrea et al., 1996). HCV genotyping was performed with a modified ELISA thybridization method (Viazoz et al., 1994) using the amplified nested PCR product of the HCV core region with digoxigenin incorporation and specific probes for HCV genotypes $1 \mathrm{a}$, $1 b, 2 a, 2 b$, and 3 as described elsewhere (Larrea et al., 1996; Sarobe et al., 1996).

Hepatitis G virus (HGV) RNA was analyzed in serum by a PCR-based procedure using a commercial kit (Boehringer Mannheim, Mannheim, Germany). This method consists of a PCR amplification after RNA extraction of two regions ( $5^{\prime}$ noncoding region and NS5a) of HGV followed by capture of digoxigeninlabeled PCR products on plated specific probes and detection using anti-digoxigenin peroxidase-labeled antibody. Sera were considered negative when the two $P C R$ products were negative.

\section{ACKNOWLEDGMENTS}

We thank Celia Asensio, Edurne Elizalde, and Beatriz Carte for assistance with the PCR assays; Dr. Iosu Sola for histological analysis; Isabel Ordoqui for technical assistance; Dr. Ana Cristina Villaro and Blanca Irigoyen for assistance in electron microscopy analysis; The Department of Radiotherapy of the University Clinic of Navarra; and finally the personnel of the animal facilities of CIFA-University of Navarra for the care of tupaias, especially Gabriel de Biurrun and Beatriz Landa. We also thank the National Science Foundation of China for support.

\section{REFERENCES}

Abe, K., Kurata, T., Teramoto, Y., Shiga, J., and Shikata, T. (1993). Lack of susceptibility of various primates and woodchucks to hepatitis C virus. J. Med. Primatol. 22, 433 \pm 434 .
Alter, H. J., Purcell, R. H., Holland, P. V., and Popper, H. (1978). Transmissible agent in non-A, non-B hepatitis. Lancet $1,459 \pm 463$.

Alter, M. J., Hadler, S. C., Judson, F. N., Mares, A., Alexander, W. J., Hu, P. Y., M iller, J. K., M oyer, L. A., Fields, H. A., Bradley, D. W. et al. (1990). Risk factors for acute non-A non-B hepatitis in the United States and association with hepatitis $C$ virus infection. J. Am. Med. Assoc. 264, $2231 \pm 2235$.

Bradley, D. W., Cook, E. H., Maynard, J. E., McCaustland, K. A., Ebert, J. W., Dolana, G. H., Petzel, R. A., Kantor, R. J., Heilbrunn, A., Fields, H. A., and Murphy, B. L. (1979). Experimental infection of chimpanzees with anti-hemophilic (factor VIII) materials: Recovery of viruslike particles associated with non-A, non-B hepatitis. J. Med. Virol. 3, $253 \pm 269$.

Bradley, D. W., M aynard, J. E., Popper, H., Ebert, J. W., Cook, E. H., Fields, H. A., and Kemler, B. J. (1981). Persistent non-A, non-B hepatitis in experimentally infected chimpanzees. J. Infect. Dis. 143, 210 \pm 218.

Bradley, D. W., Krawczynski, K., Ebert, J. W., McCaustland, K. A., Choo, Q. L., Houghton, M. A., and Kuo, G. (1990). Parenterally transmitted non-A, non-B hepatitis: Virus-specific antibody response patterns in hepatitis C virus-infected chimpanzees. Gastroenterology 99, 1054 \pm 1060.

Choo, Q. L., Kuo, G., Weiner, A. J., Overby, L. R., Bradley, D. W., and Houghton, M. (1989). Isolation of a cDNA clone derived from a blood-borne non-A, non-B viral hepatitis genome. Science $244,359 \pm$ 362

Darai, G., Schwaier, A., Komitowski, D., and Munk, K. (1978). experimental infection of Tupaia belangari (tree shrews) with herpes simplex virus type I and II. J. Infect. Dis. 137, 221 +226 .

Farci, P., Alter, H. J., Wong, D., Miller, R. H., Shih, J. W., Jett, B., and Purcell, R. H. (1991). A long-term study of hepatitis $C$ virus replication in non-A non-B hepatitis. N. Engl. J. Med. 325, 98+104.

Gavier, B., M artổez-GonzÂNez, M. A., Riezu-Boj, J. I., Lasarte, J. J., Garcia, N., Civeira, M. P., and Prieto, J. (1997). Viremia after one month of interferon therapy predicts treatment outcome in chronic hepatitis $C$. Gastroenterology 113, 1647 \pm 1653 .

Gil, B., Qian, C., Riezu-Boj, J. I., Civeira, M. P., and Prieto, J. (1993). Hepatic and extrahepatic HCV RNA strands in chronic hepatitis C: Different patterns of response to interferon treatment. Hepatology 18 , $1050 \pm 1054$.

Larrea, E., Garcia, N., Qian, C., Civeira, M. P., and Prieto, J. (1996). Tumor necrosis factor a gene expression and the response to interferon in chronic hepatitis C. Hepatology 23, 210+217.

Li, Q., Ding, M., and Wang, H. (1995). The infection of hepatitis D virus in adult tupaia. Chung. Hua. I. Hsueh. Tsa. Chih. 75, 611 \pm 613 .

Martin, R. D. (1990). Are tree-shrews primates? In "Primate Origins and Evolution: A Phylogenetic Reconstruction" (R. D. Martin, Ed.), pp. $191 \pm 213$. Chapman \& Hall, London.

Sarobe, P., Jauregui, J. I., Lasarte, J. J., Garcia, N., Civeira, M. P., Borras-Cuesta, F., and Prieto, J. (1996). Production of interleukin-2 in response to synthetic peptides from hepatitis $C$ virus $E 1$ protein in patients with chronic hepatitis $C$ : Relationship with the response to interferon treatment. J. Hepatol. 25, $1 \pm 9$.

Schreier, E., Roggendorf, M., Driesel, G., Hohne, M., and Viazov, S. (1996). Genotypes of hepatitis C virus isolates from different parts of the world. Arch. Virol. Suppl. 11, 185 \pm 193.

Shimizu, Y. K., Feinstone, S. M., Purcell, R. H., Alter, H. J., and London, W. T. (1979). Non-A, non-B hepatitis: Ultrastructural evidence for two agents in experimentally infected chimpanzees. Science $205,197 \pm$ 200.

Shindo, M m, Di Biscegliem A. M., Biswas, R., Mihalic, K., and Feinston, S. M. (1992). Hepatitis $C$ virus replication during acute infection in the chimpanzee. J. Infect. Dis. 166, 424 \pm 427.

Viazoz, S., Zibert, A., Ramakrishnan, K., Widell, A., Cavicchini, A., Schreier, E., Roggendorf, M. (1994). Typing of HCV isolates by DNA enzyme immunoassay. J. Virol. Methods 48, 81+91.

Walter, E., Keist, R., Niederost, B., Pult, I., and Blum, H. E. (1996). 
Hepatitis B virus infection of tupaia hepatocytes in vitro and in vivo. Hepatology 24, $1 \pm 5$.

Wan, B. X., Liu, J. C., Xi, O. Y., Zhang, X. S., Zhang, S. C., Wang, Z. M., Zhang, W. F., Xui, W. M., Wangm F. N., and Ding, X. F. (1982). Experimental study on the infection of adult tree shrew (Tupaia belangeri yunalis) with human rotavirus. Natl. Med. J. Chin. 62, 461 \pm 465 .

Wang, Y. X., Li, C. Y., and Ma, S. L. (1991). The classification and ecology of Chinese tree shrews. In "Biology of Chinese Tree Shrews (Tupaia belangeri chinensis)" (Y. Z. Peng, Z. Z. Ye, R. J. Zhou, Y. X. Wang, B. P. Tian, Y. Y. Ma, L. M. Shi, Eds.), pp. 21 \pm 70 . Yunnan Sci. and Technol. Press, Kunming, China.

Wu, X. X., Tang, E. H., Xie, G. Z., Zhang, X. S., Xui, W. M., Lao, Q. S., Liu, M. Y., Wen, Y. L., Zhu, B. C., and Lu, Y. J. (1983). Investigation on herpes virus of tree shrews. Chin. J. Micro. Immunol. 3, 33 \pm 36 .

Yan, R. Q., Su, J. J., Huang, D. R., Gan, Y. C., Yang, C., and Huang, G. H. (1996a). Human hepatitis B virus and hepatocellular carcinoma. I. Experimental infection of tree shrews with hepatitis B virus. J. Cancer Res. Clin. Oncol. 122, 283 \pm 288 .

Yan, R. Q., Su, J. J., Huang, D. R., Gan, Y. C., Yang, C., and Huang, G. H. (1996b). Human hepatitis B virus and hepatocellular carcinoma. II. Experimental induction of hepatocellular carcinoma in tree shrews exposed to hepatitis B virus and aflatoxin b1. J. Cancer Res. Clin. Oncol. 122, 289+295.

Zan, M. Y., Liu, C. B., Li, C. M., Zhang, W. Y., Zhu, C., Pang, Q. F., Zhao, T. X., Wang, G., and Wang, J. L. (1981). A preliminary study of hepatitis $A$ virus infection in Chinese tupaia. Acta Acad. Med. Sin. 3, $148 \pm 152$.

Zhang, W. H., Liu, C. B., Sun, Y. D., Alter, H. J., and Shih, J. W. (1990). Hepatitis $C$ virus causing non- $A$, non- $B$ hepatitis in plasmapheresis center. Lancet 335, 353. 